\title{
Pseudospin symmetry as a relativistic dynamical symmetry in the nucleus
}

\author{
P. Alberto and M. Fiolhais \\ Departamento de Física and Centro de Física Computacional, \\ Universidade de Coimbra, P-3004-516 Coimbra Portugal \\ M. Malheiro and A. Delfino \\ Instituto de Física, Universidade Federal Fluminense, \\ 24210-340 Niterói, Brazil \\ M. Chiapparini \\ Departamento de Física Teórica, \\ Universidade do Estado do Rio de Janeiro, \\ 20550-900 Rio de Janeiro, Brazil
}

(Dated: November 3, 2018)

\begin{abstract}
Pseudospin symmetry in nuclei is investigated by solving the Dirac equation with Woods-Saxon scalar and vector radial potentials, and studying the correlation of the energy splittings of pseudospin partners with the nuclear potential parameters. The pseudospin interaction is related to a pseudospin-orbit term that arises in a Schroedinger-like equation for the lower component of the Dirac spinor. We show that the contribution from this term to the energy splittings of pseudospin partners is large. The near pseudospin degeneracy results from a significant cancelation among the different terms in that equation, manifesting the dynamical character of this symmetry in the nucleus. We analyze the isospin dependence of the pseudospin symmetry and find that its dynamical character is behind the different pseudospin splittings observed in neutron and proton spectra of nuclei.
\end{abstract}

PACS numbers: 21.10.-k, 21.10.Hw, 21.60.Cs

\section{INTRODUCTION}

The idea of pseudospin was introduced to explain the quasi-degeneracy in some nuclei between single-nucleon states with quantum numbers $(n, \ell, j=\ell+1 / 2)$ and $(n-1, \ell+2, j=\ell+3 / 2)$ where $n, \ell$, and $j$ are the radial, the orbital, and the total angular momentum quantum numbers, respectively. These levels have the same "pseudo" orbital angular momentum quantum number, $\tilde{\ell}=\ell+1$, and "pseudo" spin quantum number, $\tilde{s}=1 / 2$. For example, for $\left[n s_{1 / 2},(n-1) d_{3 / 2}\right]$ one has $\tilde{\ell}=1$, for $\left[n p_{3 / 2},(n-1) f_{5 / 2}\right]$ one has $\tilde{\ell}=2$, etc. Pseudospin symmetry is exact when doublets with $j=\tilde{\ell} \pm \tilde{s}$ are degenerate.

Since the proposal of the pseudospin concept, about 30 years ago [1, 2, the underlying mechanism of pseudospin symmetry in nuclei has become a topic of intense theoretical research. In a recent letter [3], we have shown the dynamical character of this symmetry in nuclei, arising from balance effects among the central nuclear potentials parameters. We have also concluded that this dynamical behavior of the symmetry is the main explanation for the isospin asymmetry of the nuclear pseudospin orbit interaction observed in nature. In this paper we elaborate on the main topics of that letter, extending our analysis for different nuclei.

It is worth reviewing the main results of several papers [4, 5, 6, 月, 8, 90 in which the possible underlying mechanisms to generate pseudospin symmetry have been discussed.

A helicity unitary transformation of a non-relativistic single-particle Hamiltonian was considered by Blokhin et al. [1. They showed that the transformed radial wave functions behave as

$$
\tilde{R}_{n j \tilde{\ell}} \sim \begin{cases}r^{\tilde{\ell}}, & r \rightarrow 0 \\ r^{-(\ell+3)}, & r \rightarrow \infty\end{cases}
$$

Moreover, they claimed this behavior to be universal and conjectured that, at small distances, the helicity transformed mean field of a heavy nucleus should not be much different from the untransformed one, since the behavior of $\tilde{R}$ remains similar to $R$ near the origin. At larger distances, however, as suggested by the above asymptotic behavior of the transformed wave function, the helicity transformed mean field would acquire a more diffuse surface. At the same time, the helicity transformed fields would get strongly nonlocal at the surface. The application of the helicity operator to the non-relativistic single-particle wave function maps the normal state $(\ell, s)$ onto the "pseudo" state $(\tilde{\ell}, \tilde{s})$, while keeping all other global symmetries 4 .

A similar kind of unitary transformation was also considered earlier to discuss the pseudospin symmetry in the nonrelativistic harmonic oscillator [5. 6]. It was shown that, if the ratio of the coefficients of spin-orbit and orbit-orbit 
terms in the single-particle spherical Nilsson Hamiltonian has a particular value, pseudospin symmetry will be exact. Bahri et al. [5] showed that this value was approached by relativistic mean field (RMF) estimates for that ratio.

More recently, the subject was revived when Ginocchio 7 recognized, for the first time, the relativistic character of the symmetry. He noted that the pseudo-orbital angular momentum is just the orbital angular momentum of the lower component of the Dirac spinor. He also showed that pseudospin symmetry is an exact symmetry for the Dirac Hamiltonian with an attractive scalar potential, $S$, and a repulsive vector potential $V$, when these potentials are equal in magnitude: $S+V=0$. It turns out that this symmetry is nothing but a $\mathrm{SU}(2)$ symmetry of the Dirac Hamiltonian [10, 11, 12].

In RMF models, often referred to as Quantum Hadrodynamics 13, the nuclear saturation mechanism is explained by the cancelation between a large scalar and a large vector fields [13, 14, 15, 16, 17]. Typical values for these fields in heavy nuclei are of the order of a few hundred $\mathrm{MeV}$ (with opposite signs), their sum providing a binding potential of about $-60 \mathrm{MeV}$ at the center. Therefore, it was natural for Ginocchio to regard the quasi-degenerate pseudospin doublets in nuclei as arising from the near equality in magnitude of the attractive scalar, $S$, and the repulsive vector, $V$, relativistic mean fields, $S \sim-V$, in which the nucleons move.

After the work of Ginocchio, Meng et al. 8, 9] showed that pseudospin symmetry is exact when $d \Sigma / d r=0$, where $\Sigma=S+V$. They also related the onset of the pseudospin symmetry as a competition between the centrifugal barrier and the pseudospin-orbit potential. In short, they claimed that the following inequality assures pseudospin symmetry:

$$
\frac{\kappa}{r} \frac{d \Sigma(r)}{d r} \ll[E-\Sigma(r)] \frac{\kappa(\kappa-1)}{r^{2}},
$$

where the quantum number $\kappa$ obeys $\kappa(\kappa-1)=\ell(\ell+1)$ and $E$ is the binding energy. The above inequality is, of course, satisfied in the particular cases $\Sigma=0$ and $d \Sigma / d r=0$. One may note that the latter condition implies the former if $S, V \rightarrow 0$ at large distances, which is the case of the nucleus. However, this criterium presents several practical problems that are not easily overcome [18], namely it cannot be applied in the range of values of $r$ for which $E \sim \Sigma(r)$. This is related to the fact that the pseudospin-orbit term has the denominator $E-\Sigma(r)$ and thus becomes infinite when $E=\Sigma(r)$ [8, 9, 18].

In other works [19, 20,21, 22, 23] the pseudospin symmetry has also been discussed in relation with the arguments and conjectures of refs. [7, 8, 9. Recently, a test of nuclear wave functions for pseudospin symmetry was done in [24. In a more recent work, the structure of radial nodes occuring in pseudospin levels and a classification for the intruder levels (states which do not have a pseudospin partner in the limit of pseudospin symmetry) was explained as a direct effect of the behavior of nodes of Dirac bound states [25], giving support for the relativistic interpretation of nuclear pseudospin symmetry. However, despite these reports on the quasi-degeneracy of pseudospin doublets, the nature of pseudospin breaking in nuclei is not fully understood.

In this paper we address this question by studying the splittings of the neutron single-particle energy levels for pseudospin partners obtained by solving the Dirac equation with vector and scalar potentials of Woods-Saxon type. From the behavior of these splittings with Woods-Saxon parameters, we are able to show that pseudospin symmetry has a dynamical character. As we have already pointed out, the present paper is a natural extension of [3], providing more technical details and complementary physical information. In addition, we calculate the expectation value of the pseudospin-orbit coupling and find that it correlates well with the observed pseudospin splittings.

The paper is organized as follows: in Section II we review the formalism of the Dirac equation with scalar and vector potentials, its relation to pseudospin symmetry and identify the pseudospin-orbit term. The neutron energy levels and respective pseudospin splittings obtained in a relativistic calculation with Woods-Saxon mean fields are presented in Section III. We consider the cases of ${ }^{208} \mathrm{~Pb},{ }^{40} \mathrm{Ca}$ and ${ }^{48} \mathrm{Ca}$ and draw the systematics for the variation of pseudospin splittings with the Woods-Saxon potential parameters. We also study the contribution to the energy splitting of pseudospin partners by each term in the Schroedinger-like second order differential equation for the lower component of the Dirac spinor, in particular the contribution of the pseudospin-orbit term. Using this systematics, in Section IV we explain the differences of those splittings for neutrons and protons and why this does not happen with spin-orbit splittings. In Section $\mathrm{V}$ we give a brief summary of our main conclusions.

\section{DIRAC EQUATION AND PSEUDOSPIN SYMMETRY}

The Hamiltonian of a Dirac particle of mass $m$ in an external scalar, $S$, and vector, $V$, potentials is given by

$$
H=\boldsymbol{\alpha} \cdot \boldsymbol{p}+\beta(m+S)+V,
$$

where $\boldsymbol{\alpha}$ and $\beta$ are the Dirac matrices

$$
\beta=\left(\begin{array}{cc}
I & 0 \\
0 & -I
\end{array}\right), \quad \boldsymbol{\alpha}=\left(\begin{array}{cc}
0 & \boldsymbol{\sigma} \\
\boldsymbol{\sigma} & 0
\end{array}\right),
$$


and $\boldsymbol{\sigma}$ are the Pauli matrices.

The Hamiltonian (3) is invariant under $\mathrm{SU}(2)$ transformations for two cases: $S=V$ and $S=-V$ 11, 12]. The first case was recently invoked to explain the spectrum of some mesons, exhibiting small spin-orbital splittings 26 . The second situation would lead to exact pseudospin symmetry in nuclei, with $\Sigma=0$. However, since $\Sigma$ plays the role of a binding potential, this value for $\Sigma$ would imply that there would be no bound nucleon states [7]. In relativistic mean field models the attractive scalar and the repulsive vector parts of the nucleon potential are similar in magnitude but they are not equal, and so that limiting case is never reached.

We define $\Delta=V-S$ and denote the upper and lower components of the Dirac spinor by $\Psi_{ \pm}=\frac{1 \pm \beta}{2} \Psi$. The Dirac equation $H \Psi=\epsilon \Psi$ with $H$ given by (3) and energy $\epsilon=E+m$ gives rise to two coupled first-order differential equations for the upper and lower components:

$$
\begin{aligned}
& \boldsymbol{\alpha} \cdot \boldsymbol{p} \Psi_{-}=(E-\Sigma) \Psi_{+} \\
& \boldsymbol{\alpha} \cdot \boldsymbol{p} \Psi_{+}=(E+2 m-\Delta) \Psi_{-} .
\end{aligned}
$$

If one multiplies these equations by $\boldsymbol{\alpha} \cdot \boldsymbol{p}$ one gets

$$
\begin{aligned}
& p^{2} \Psi_{-}=-[\boldsymbol{\alpha} \cdot \boldsymbol{p} \Sigma] \Psi_{+}+(E-\Sigma) \boldsymbol{\alpha} \cdot \boldsymbol{p} \Psi_{+} \\
& p^{2} \Psi_{+}=-[\boldsymbol{\alpha} \cdot \boldsymbol{p} \Delta] \Psi_{-}+(E+2 m-\Delta) \boldsymbol{\alpha} \cdot \boldsymbol{p} \Psi_{-},
\end{aligned}
$$

where the square brackets mean that the operator $\boldsymbol{\alpha} \cdot \boldsymbol{p}$ only acts on the potential in front of it. Using Eqs. (5) one obtains

$$
\begin{aligned}
& p^{2} \Psi_{-}=-[\boldsymbol{\alpha} \cdot \boldsymbol{p} \Sigma] \Psi_{+}+(E+2 m-\Delta)(E-\Sigma) \Psi_{-} \\
& p^{2} \Psi_{+}=-[\boldsymbol{\alpha} \cdot \boldsymbol{p} \Delta] \Psi_{-}+(E+2 m-\Delta)(E-\Sigma) \Psi_{+} .
\end{aligned}
$$

Assuming that the potentials $\Sigma$ and $\Delta$ are radial functions, and using again Eqs. (5), we obtain the following two decoupled second-order differential equations for $\Psi_{ \pm}$:

$$
\begin{aligned}
& p^{2} \Psi_{-}=-\frac{\Sigma^{\prime}}{E-\Sigma}\left(-\frac{\partial}{\partial r}+\frac{1}{r} \boldsymbol{\sigma} \cdot \boldsymbol{L}\right) \Psi_{-}+(E+2 m-\Delta)(E-\Sigma) \Psi_{-} \\
& p^{2} \Psi_{+}=-\frac{\Delta^{\prime}}{E+2 m-\Delta}\left(-\frac{\partial}{\partial r}+\frac{1}{r} \boldsymbol{\sigma} \cdot \boldsymbol{L}\right) \Psi_{+}+(E+2 m-\Delta)(E-\Sigma) \Psi_{+}
\end{aligned}
$$

where the primes denote derivatives with respect to $r$. The spinors $\Psi_{ \pm}$can be factorized into radial and angular parts: $\Psi_{+}=\mathrm{i} G_{i}(r) \Phi_{i}^{+}(\theta, \phi)$ and $\Psi_{-}=-F_{i}(r) \Phi_{i}^{-}(\theta, \phi)$, where $i$ denotes the quantum numbers of the single particle state. In terms of the radial functions $G_{i}$ and $F_{i}$ for upper and lower components, these equations become

$$
\begin{array}{r}
\nabla^{2} F_{i}+\frac{\Sigma^{\prime}}{E-\Sigma}\left(F_{i}^{\prime}+\frac{1-\kappa_{i}}{r} F_{i}\right)+(E+2 m-\Delta)(E-\Sigma) F_{i}=0 \\
\nabla^{2} G_{i}+\frac{\Delta^{\prime}}{E+2 m-\Delta}\left(G_{i}^{\prime}+\frac{1+\kappa_{i}}{r} G_{i}\right)+(E+2 m-\Delta)(E-\Sigma) G_{i}=0,
\end{array}
$$

where the property $\boldsymbol{\sigma} \cdot \boldsymbol{L} \Phi_{i}^{ \pm}=-\left(1 \pm \kappa_{i}\right) \Phi_{i}^{ \pm}$was used. The $\boldsymbol{\sigma} \cdot \boldsymbol{L}$ terms in the equations for $F_{i}$ and $G_{i}$ are related to pseudospin-orbital and spin-orbital couplings respectively [8, 9, 23].

Let us look more closely at the Eq. (10) for $\Psi_{-}$. The denominator $E-\Sigma$ in the $\boldsymbol{\sigma} \cdot \boldsymbol{L}$ term comes from the replacement of $\Psi_{+}$, using Eq. (5a), in the term $-[\boldsymbol{\alpha} \cdot \boldsymbol{p} \Sigma] \Psi_{+}$of Eq. (8), since

$$
\Psi_{+}=\frac{\boldsymbol{\alpha} \cdot \boldsymbol{p} \Psi_{-}}{E-\Sigma}=\frac{\mathrm{i} \boldsymbol{\alpha} \cdot \hat{r}}{E-\Sigma}\left(-\frac{\partial}{\partial r}+\frac{1}{r} \boldsymbol{\sigma} \cdot \boldsymbol{L}\right) \Psi_{-} .
$$

From Eq. (5a), when $E-\Sigma=0$, then $\boldsymbol{\alpha} \cdot \boldsymbol{p} \Psi_{-}=0$, meaning that, in Eq. (14), the whole term in parenthesis applied to $\Psi_{-}$gives zero. Therefore, although each of the two terms within the parenthesis, divided by $E-\Sigma$, is infinite when $E=\Sigma$, their sum is finite. The analysis of these terms is better done by rewriting Eq. (8) as a Schroedinger-like equation:

$$
\frac{p^{2}}{2 m^{*}} \Psi_{-}+\frac{[\boldsymbol{\alpha} \cdot \boldsymbol{p} \Sigma]}{2 m^{*}} \Psi_{+}+\Sigma \Psi_{-}=E \Psi_{-}
$$


where an energy- and $r$-dependent effective mass $m^{*}=(E+2 m-\Delta) / 2$ was defined. Applying again Eq. (14) we have

$$
\frac{p^{2}}{2 m^{*}} \Psi_{-}+\frac{1}{2 m^{*}} \frac{\Sigma^{\prime}}{E-\Sigma}\left(-\frac{\partial}{\partial r}+\frac{1}{r} \boldsymbol{\sigma} \cdot \boldsymbol{L}\right) \Psi_{-}+\Sigma \Psi_{-}=E \Psi_{-} .
$$

Now we can identify the various terms contributing to the binding energy $E$ and, in particular, assess the role of the term $\Sigma^{\prime} /\left[2 m^{*}(E-\Sigma)\right] \boldsymbol{\sigma} \cdot \boldsymbol{L} / r \Psi_{-}$in the pseudospin energy splitting. This is possible since the principal value of the integral of this term, after multiplying it by $\Psi_{-}^{\dagger}$, is finite. Furthermore, the following sum rule should be satisfied:

$$
-\mathrm{P} \int \Psi_{-}^{\dagger} \frac{1}{2 m^{*}} \frac{\Sigma^{\prime}}{E-\Sigma} \frac{\partial \Psi_{-}}{\partial r} \mathrm{~d}^{3} \boldsymbol{r}+\mathrm{P} \int \Psi_{-}^{\dagger} \frac{1}{2 m^{*}} \frac{\Sigma^{\prime}}{E-\Sigma} \frac{1}{r} \boldsymbol{\sigma} \cdot \boldsymbol{L} \Psi_{-} \mathrm{d}^{3} \boldsymbol{r}=\int \Psi_{-}^{\dagger} \frac{-\mathrm{i} \boldsymbol{\alpha} \cdot \hat{r} \Sigma^{\prime}}{2 m^{*}} \Psi_{+} \mathrm{d}^{3} \boldsymbol{r}
$$

where ' $\mathrm{P}$ ' denotes the principal value of the integral. The analysis of the contribution of the various terms in Eq. (16) will be done in the next section.

It is interesting to discuss how the pseudospin symmetry gets broken. The SU(2) generators of the pseudospin symmetry are given by 10, 11, 12]

$$
\begin{aligned}
S_{i} & =s_{i} \frac{1}{2}(1-\beta)+\frac{\boldsymbol{\alpha} \cdot \boldsymbol{p} s_{i} \boldsymbol{\alpha} \cdot \boldsymbol{p}}{p^{2}} \frac{1}{2}(1+\beta)= \\
& =\left(\begin{array}{cc}
\tilde{s}_{i} & 0 \\
0 & s_{i}
\end{array}\right)
\end{aligned}
$$

where

$$
\tilde{s}_{i}=\frac{\boldsymbol{\sigma} \cdot \boldsymbol{p}}{p} s_{i} \frac{\boldsymbol{\sigma} \cdot \boldsymbol{p}}{p}=\frac{2 s \cdot \boldsymbol{p}}{p^{2}} p_{i}-s_{i}
$$

and $s_{i}=\sigma_{i} / 2(i=1,2,3)$. The commutator of this operator with the Hamiltonian (3) is

$$
\left[H, S_{i}\right]=\left(\begin{array}{cc}
{\left[\Sigma, \tilde{s}_{i}\right]} & 0 \\
0 & 0
\end{array}\right)
$$

Thus, the pseudospin symmetry breaking can be related to the commutator $\left[\Sigma, \tilde{s}_{i}\right]$. If we take the non-relativistic limit, we recover the findings of Ref. [1 as long as $\tilde{s}_{i}$ commutes with the non-relativistic Hamiltonian $H_{n r}$. This is equivalent to consider the helicity transformed spin operator, $\tilde{s}_{i}$, to be the generator of a $\mathrm{SU}(2)$ symmetry of the non-relativistic Hamiltonian, $\left[H_{n r}, \tilde{s}_{i}\right]=0$, implying $\left[\tilde{H}_{n r}, s_{i}\right]=0$, where

$$
\tilde{H}_{n r}=\frac{\boldsymbol{\sigma} \cdot \boldsymbol{p}}{p} H_{n r} \frac{\boldsymbol{\sigma} \cdot \boldsymbol{p}}{p}
$$

is the helicity transformed non-relativistic Hamiltonian of Ref. 幽. Requiring $\left[\Sigma, \tilde{s}_{i}\right]=0$ is equivalent to the previous conditions $\Sigma=S+V=0$ or $d \Sigma / d r=0$, when $\Sigma$ is a radial function $[7,8,9$.

\section{PSEUDOSPIN ENERGY SPLITTINGS FOR WOODS-SAXON MEAN FIELDS}

As stated above, the equivalent conditions $\Sigma=S+V=0$ and $d \Sigma / d r=0$ are not met in nuclei. From Ginocchio's findings [7], one expects that there is a correlation between the pseudospin splitting and the $\Sigma$ depth. Our purpose here is to go further and investigate whether there is also a correlation between those splittings and the shape of the mean-field nuclear potential, namely its radius and surface diffuseness.

To this end, we perform a model calculation using a Lorentz structured relativistic potential of Woods-Saxon type in the Dirac equation. The scalar and vector components of this potential are the mean-field central nuclear potentials. The potentials are given by

$$
U(r)=\frac{U_{0}}{1+\exp [(r-R) / a]},
$$

where $U(r)$ stands either for the vector or for the scalar potential. Although this potential is not a full self-consistent relativistic potential derived from meson fields, it is realistic enough to be applied to nuclei. Indeed, most selfconsistent potentials have a Woods-Saxon-like shape, i.e., one can recognize in them a depth, $U_{0}$, a radius (range), 
$R$, and a diffusivity, $a$. Therefore, the study of pseudospin partners splittings as a function of these parameters is meaningful and realistic enough to be applied to most nuclei, at least qualitatively. It is known that, in certain isotope chains, the central depth and the surface diffuseness changes sensibly [27, 28]. At the same time, as the mass number $A$ increases, the nuclear radius increases according to the $A^{1 / 3}$ law, which means that it is also important to study the role of the parameter $R$ in pseudospin symmetry.

Using Woods-Saxon potentials for $\Sigma$ and $\Delta$, we solved numerically the coupled first-order Dirac equations for the radial fields $F_{i}(r)$ and $G_{i}(r)$ obtained by factoring out the angular wave functions of $\Psi_{ \pm}$in Eqs. (5). They read

$$
\begin{aligned}
F_{\tilde{\ell}}^{\prime}+\frac{1+\kappa_{\tilde{\ell}}}{r} F_{\tilde{\ell}} & =-(E-m-\Sigma) G_{\ell} \\
G_{\ell}^{\prime}+\frac{1+\kappa_{\ell}}{r} G_{\ell} & =(E+m-\Delta) F_{\tilde{\ell}}
\end{aligned}
$$

where

$$
\begin{aligned}
& \kappa_{\ell}=\left\{\begin{array}{cc}
-(\ell+1) & j=\ell+\frac{1}{2} \\
\ell & j=\ell-\frac{1}{2}
\end{array}\right. \\
& \kappa_{\tilde{\ell}}=-\kappa_{\ell}=\left\{\begin{array}{cc}
-(\tilde{\ell}+1) & j=\tilde{\ell}+\frac{1}{2} \\
\tilde{\ell} & j=\tilde{\ell}-\frac{1}{2}
\end{array} .\right.
\end{aligned}
$$

There are altogether six parameters for $\Sigma$ and $\Delta$, namely the central depths, $\Sigma_{0}$ and $\Delta_{0}$, two radii and two diffuseness parameters. We observed that the pseudospin splitting is not sensitive to $R$ and $a$ of the $\Delta$ potential, and, accordingly, set the same radius, $R$, and surface diffuseness, $a$, for both potentials. We first fit these parameters to the neutron spectra of ${ }^{208} \mathrm{~Pb}$. The quality of our fitting is showed in Fig. 1, where the results of the present calculation (WS) are shown, together with the experimental values and those obtained using a relativistic mean field approach (model G1 of Ref. 29]) for the same set of pseudospin doublets: $\left(1 i_{11 / 2}, 2 g_{9 / 2}\right),\left(2 f_{5 / 2}, 3 p_{3 / 2}\right)$ and $\left(1 h_{9 / 2}, 2 f_{7 / 2}\right)$, which are the 3 topmost pseudospin partner levels in ${ }^{208} \mathrm{~Pb}$.

\section{${ }^{208} \mathrm{~Pb}$ neutrons}

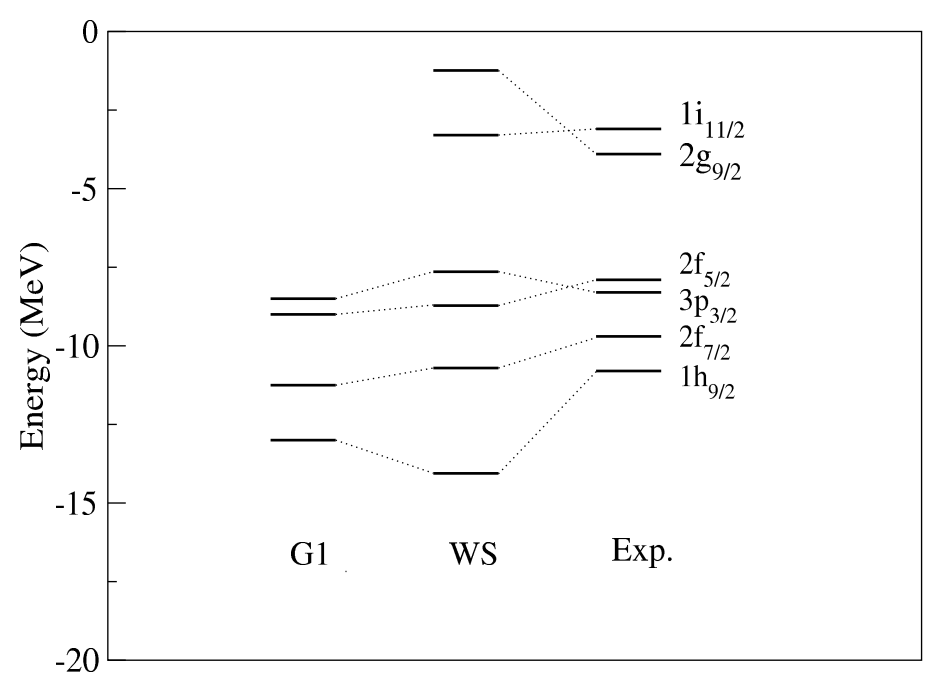

FIG. 1: Calculated neutron single-particle energy levels of the pseudospin partners $\left(1 h_{9 / 2}, 2 f_{7 / 2}\right),\left(2 f_{5 / 2}, 3 p_{3 / 2}\right)\left(1 i_{11 / 2}, 2 g_{9 / 2}\right)$ in ${ }^{208} \mathrm{~Pb}$ (WS). The leftmost values are the corresponding values of the model G1 of Furnsthal et al. 29. and the experimental values are in the rightmost column. The parameters for the Woods-Saxon potential used to fit the ${ }^{208} \mathrm{~Pb}$ neutron energy levels are $R=7 \mathrm{fm}, \Delta_{0}=650 \mathrm{MeV}, \Sigma_{0}=-66 \mathrm{MeV}$ and $a=0.6 \mathrm{fm}$. The experimental values were taken from Ref. 30].

The parameters used for the fit are given in the caption of Fig. 1. For the present purpose, our fitting is quite reasonable. Keeping $\Sigma_{0}, \Delta_{0}$, and $R$ fixed, we vary $a$ in order to see how the energies of the pseudospin doublets are sensitive to the surface diffuseness. This dependence is shown in Fig. 2. As $a$ increases, the splittings of the pseudospin doublets decrease. This could be expected because the derivative of $\Sigma$ decreases when $a$ increases. However, by further increasing $a$, the pseudospin doublet partners cross each other, inverting the sign of the energy splitting. This 
inversion of pseudospin partner splittings, $E_{n-1, \tilde{l}+1 / 2}<E_{n, \tilde{l}-1 / 2}$ changing to $E_{n-1, \tilde{l}+1 / 2}>E_{n, \tilde{l}-1 / 2}$, is observed experimentally and was also found in [9, 18, 19. It also occurs for calcium isotopes, analyzed below.

It is important to note that, once the pseudospin doublet partners cross each other, i.e., the pseudospin splitting changes sign, the effect of increasing $a$ is to drive the pseudospin doublets further apart. This systematics is consistent with the non-relativistic prediction of Ref. [国] as shown by the asymptotic behavior of the radial wave function (11).

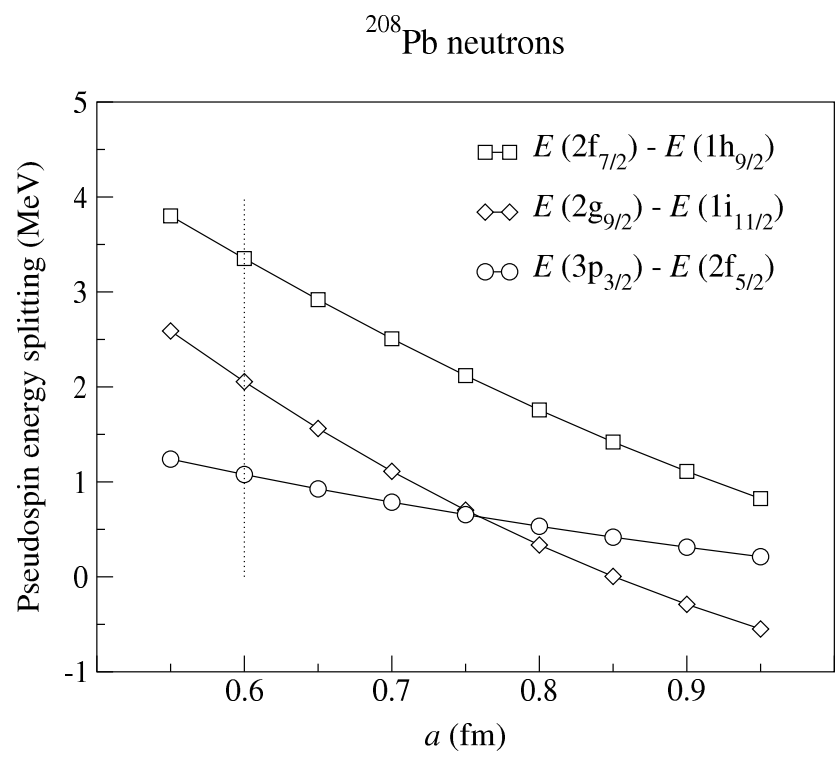

FIG. 2: pseudospin energy splittings in ${ }^{208} \mathrm{~Pb}$ as a function of the diffusivity for the neutron pseudospin partners of Fig. 1. The vertical line corresponds to the fitted Woods-Saxon parameters for ${ }^{208} \mathrm{~Pb}$ given in the caption of Fig. 1.

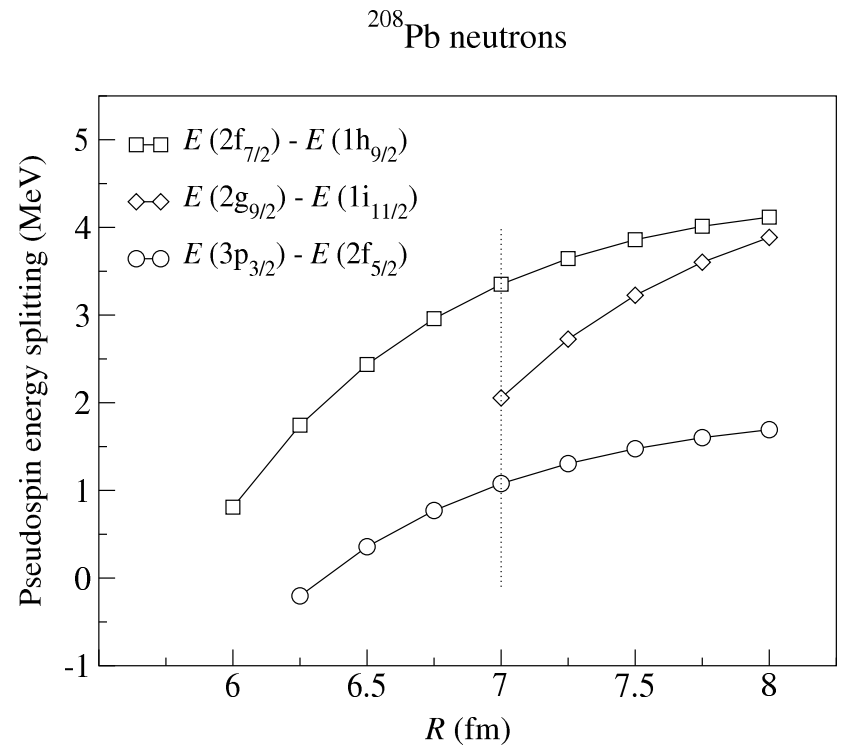

FIG. 3: pseudospin energy splittings in ${ }^{208} \mathrm{~Pb}$ as a function of the radius for the same neutron pseudospin partners of Fig. 1. The vertical line corresponds to the fitted Woods-Saxon parameters for ${ }^{208} \mathrm{~Pb}$ given in the caption of Fig. 1.

A similar trend is seen when we let the radius $R$ vary, fixing all other parameters. The result is shown in Fig. 3 . When the radius increase, the energy splittings increase, which again can be understood by the dependence of $d \Sigma / d r$ with $R$. However, we found that the dependence of the pseudospin splitting on $R$ is the opposite for the deepest energy levels, which means there are also some surface effects in this trend. This does not happen when the diffusivity changes, the trend being the same for all levels. Here we can see again the phenomenon of level crossing for the 
pseudospin partners $\left(2 f_{5 / 2}, 3 p_{3 / 2}\right)$. For $R<7 \mathrm{fm}$ we could not get bound solutions for one or both $1 i_{11 / 2}, 2 g_{9 / 2}$ levels. This dependence on $R$ is especially important when comparing different isotopes, since, in many cases, it is the radius of the mean-field potentials that changes most noticeably. Finally, we keep $a, R$ and $\Delta_{0}$ fixed but vary $\Sigma_{0}$ in order to study the sensitiveness of the pseudospin doublets with the depth of the central $\Sigma$ mean field potential. The results are presented in Fig. 4 and the following behavior is observed: as $\left|\Sigma_{0}\right|$ decreases, the splitting also decreases. This is in accordance with Ginocchio predictions for pseudospin symmetry breaking due to the finiteness of the $\Sigma$ mean field.

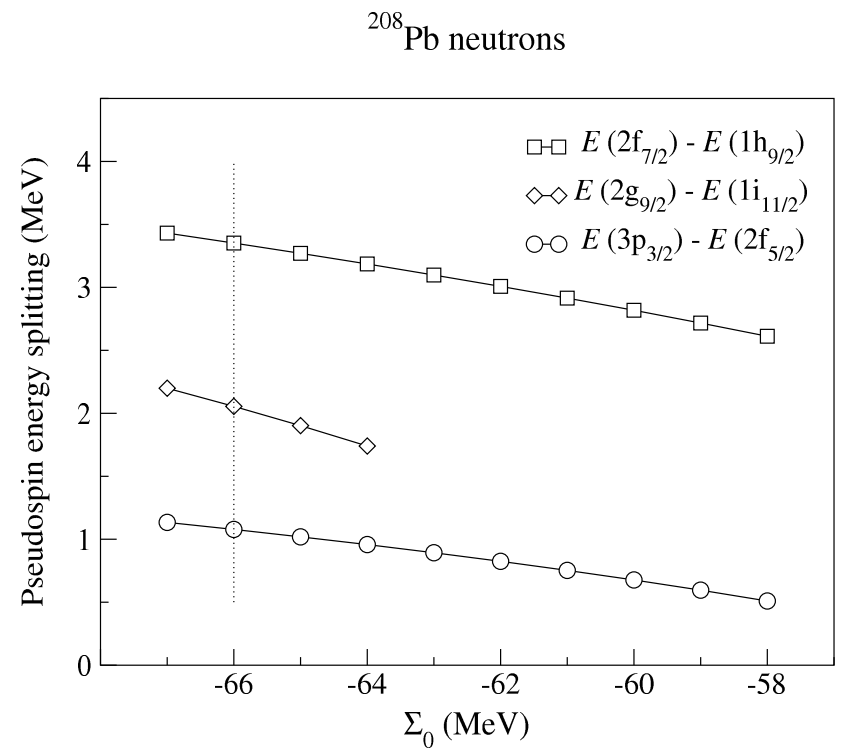

FIG. 4: Pseudospin energy splitting in ${ }^{208} \mathrm{~Pb}$ as a function of the depth of the $\Sigma$ potential for the same neutron pseudospin partners (similar to Fig. 1). The vertical line corresponds to the fitted Woods-Saxon parameters for ${ }^{208} \mathrm{~Pb}$ given in the caption of Fig. 1.

From Fig. 4 we see that the decreasing of $\left|\Sigma_{0}\right|$ shifts the levels $\left(1 i_{11 / 2}, 2 g_{9 / 2}\right)$ to the continuum. We also found that, for a deeper pseudospin doublet, an inversion of pseudospin energy splitting occurs for sufficiently low $\left|\Sigma_{0}\right|$.

${ }^{208} \mathrm{~Pb}$ neutrons

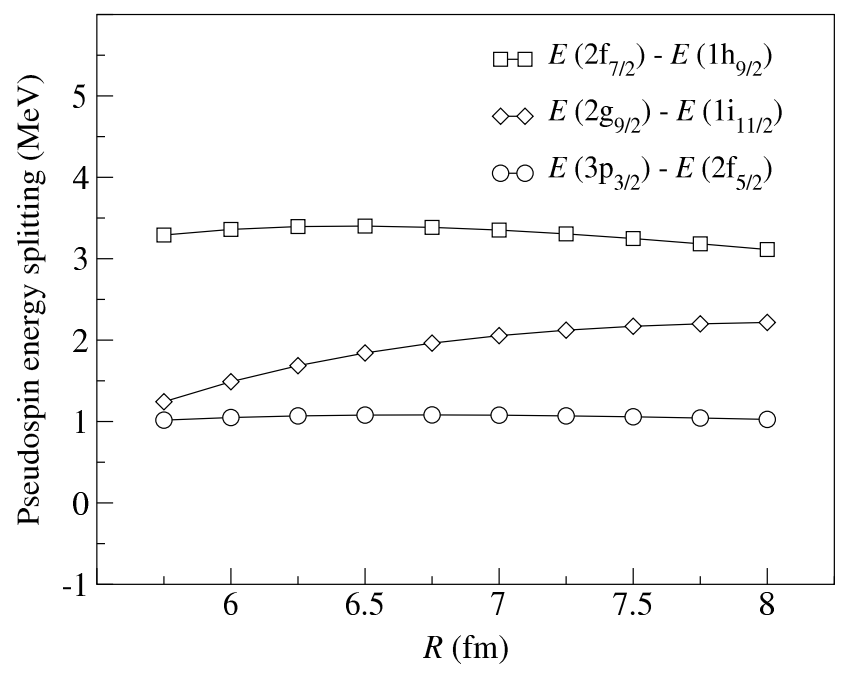

FIG. 5: Pseudospin energy splitting in ${ }^{208} \mathrm{~Pb}$ as a function of $R$ keeping $a$ and the product $\left|\Sigma_{0}\right| R^{2}$ fixed for the same neutron pseudospin partners as in Fig. 1. 
Actually, we have found that $\left|\Sigma_{0}\right|$ and $R$ are not completely independent parameters. Varying $\left|\Sigma_{0}\right|$ and $R$ but keeping $a$ and the product $\left|\Sigma_{0}\right| R^{2}$ fixed, the pseudospin splittings remain almost constant, as shown in Fig. 5 .

We observed that varying the other free parameter, $\Delta_{0}$, does not qualitatively change the splittings. Since the $\Delta$ potential is related to the effective mass of the nucleons, the basic effect is to slightly change the nucleon separation energies, and especially the spin-orbital coupling, as discussed below.

Although the Woods-Saxon potential is expected to be a good approximation for central potentials of heavy nuclei like lead, some calculations indicate that it might also be good enough for lighter nuclei such as calcium [27. Therefore, in order to show the importance of the surface diffuseness and of the radius in the dynamical pseudospin symmetry, we applied our model also to two calcium isotopes, where the pseudospin splitting is also small. We fitted our free

\begin{tabular}{|c|c|c|c|c|c|c|c|}
\hline Nucleus & $R$ & $a$ & $\Sigma_{0}$ & $\Delta_{0}$ & Level & $E_{\text {calc }}$ & $E_{\exp }$ \\
\hline \multirow{2}{*}{${ }^{40} \mathrm{Ca}$} & \multirow{2}{*}{3.8} & \multirow{2}{*}{0.8} & \multirow{2}{*}{-78} & \multirow{2}{*}{770} & $2 s_{1 / 2}$ & -16.0 & -18.2 \\
\hline & & & & & $1 d_{3 / 2}$ & -15.1 & -15.6 \\
\hline \multirow{2}{*}{${ }^{48} \mathrm{Ca}$} & \multirow{2}{*}{4.0} & \multirow{2}{*}{0.8} & \multirow{2}{*}{-64} & \multirow{2}{*}{650} & $2 s_{1 / 2}$ & -12.6 & -12.4 \\
\hline & & & & & $1 d_{3 / 2}$ & -12.2 & -12.4 \\
\hline
\end{tabular}

TABLE I: Calculated and experimental energy values of the neutron pseudospin partners $2 s_{1 / 2}$ and $1 d_{3 / 2}$ in ${ }^{40} \mathrm{Ca}$ and ${ }^{48} \mathrm{Ca}$. The Woods-Saxon potential parameters used to fit the energy levels of these nuclei are also displayed. The radius, $R$, and the diffuseness, $a$, are in fm and the energies in $\mathrm{MeV}$. The experimental values are from Ref. [3].

parameters to the experimental values of the energies of the topmost neutron levels of ${ }^{40} \mathrm{Ca}$ and ${ }^{48} \mathrm{Ca}$. The results for the pseudospin partners $\left(2 s_{1 / 2}, 1 d_{3 / 2}\right)$ are presented in Table 1 and compared with the experimental values.

The difference between the ${ }^{48} \mathrm{Ca}$ neutron potential with respect to the ${ }^{40} \mathrm{Ca}$ neutron potential lies basically in a smaller modulus of the central depth and in a larger radius. We also see that the magnitude of pseudospin energy splitting decreases from $0.9 \mathrm{MeV}$ to $0.4 \mathrm{MeV}$ as the number of nucleons $A$ increases. Since the levels $2 s_{1 / 2}$ and $1 d_{3 / 2}$ are inverted in ${ }^{40} \mathrm{Ca}$, the effect of the change in $R$ predicted by the systematics is to increase the difference $E_{2 s_{1 / 2}}-E_{1 d_{3 / 2}}$, or, since this value is negative, to decrease its magnitude, favoring the symmetry.

We are going now to examine more closely the effect of the term $\Sigma^{\prime} /\left[(E-\Sigma)\left(2 m^{*}\right)\right] \boldsymbol{\sigma} \cdot \boldsymbol{L} / r \Psi_{-}$in Eq. (16) on pseudospin energy splittings. If we multiply that equation by $\Psi_{-}^{\dagger}$, integrate, and then divide the result by $\int \Psi_{-}^{\dagger} \Psi_{-} \mathrm{d}^{3} \boldsymbol{r}$, we get

$$
\left\langle\frac{p^{2}}{2 m^{*}}\right\rangle+\left\langle V_{\mathrm{PSO}}\right\rangle+\left\langle V_{\mathrm{D}}\right\rangle+\langle\Sigma\rangle=E
$$

where

$$
\begin{aligned}
\left\langle\frac{p^{2}}{2 m^{*}}\right\rangle & =\frac{\int_{0}^{\infty} d r \frac{1}{2 m^{*}}\left[F_{\tilde{\ell}} \frac{d}{d r}\left(r^{2} \frac{d F_{\tilde{\ell}}}{d r}\right)+\tilde{\ell}(\tilde{\ell}+1) F_{\tilde{\ell}}^{2}\right]}{\int_{0}^{\infty} r^{2} d r F_{\tilde{\ell}}^{2}} \\
\left\langle V_{\mathrm{PSO}}\right\rangle & =\frac{\mathrm{P} \int_{0}^{\infty} r d r \frac{1}{2 m^{*}} \frac{\Sigma^{\prime}}{E-\Sigma}\left(\kappa_{\ell}-1\right) F_{\tilde{\ell}}^{2}}{\int_{0}^{\infty} r^{2} d r F_{\tilde{\ell}}^{2}} \\
\left\langle V_{\mathrm{D}}\right\rangle & =-\frac{\mathrm{P} \int_{0}^{\infty} r^{2} d r \frac{1}{2 m^{*}} \frac{\Sigma^{\prime}}{E-\Sigma} F_{\tilde{\ell}} \frac{d F_{\tilde{\ell}}}{d r}}{\int_{0}^{\infty} r^{2} d r F_{\tilde{\ell}}^{2}} \\
\langle\Sigma\rangle & =\frac{\int_{0}^{\infty} r^{2} d r \Sigma F_{\tilde{\ell}}^{2}}{\int_{0}^{\infty} r^{2} d r F_{\tilde{\ell}}^{2}} .
\end{aligned}
$$

These terms can be identified, respectively, as a kinetic term, a pseudospin-orbit term, a potential term related to 
what is sometimes called Darwin term and the mean value of the $\Sigma$ potential with respect to the lower component, $\Psi_{-}$. We computed numerically the principal values, checking that the sum rule (17) was satisfied.

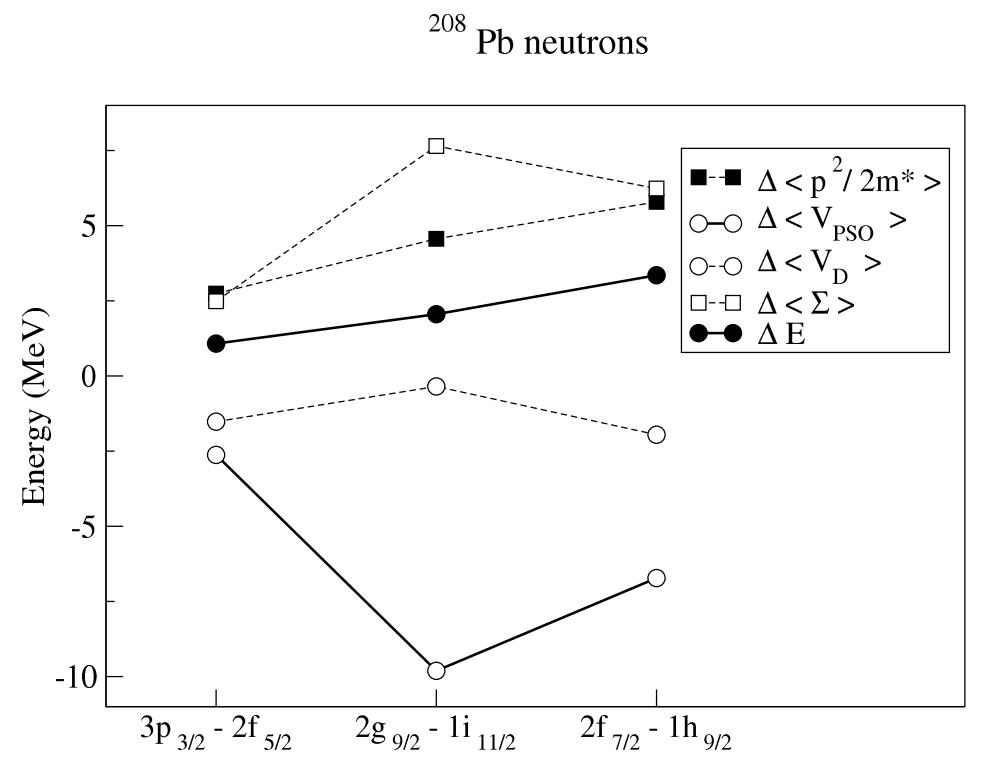

FIG. 6: Contribution of the terms (27) - 30 to the energy splittings $\Delta E$ of the pseudospin partners of Fig. 1. The strong cancelation among the terms produces a small $\Delta E$.

The results are shown in Figs. 6, 7 and 8. In Fig. 6 is plotted the difference of the terms in Eq. (26) between each member of the pseudospin partners we have been considering. One sees that the contribution for the pseudospin energy splittings of the $V_{\mathrm{PSO}}$ term is larger than the splitting itself and it is at least of the same order of magnitude and has the opposite sign of the kinetic and $\langle\Sigma\rangle$ terms. The relative contribution of each term depends on the particular pseudospin doublet we are considering. This can be seen clearly in figures 7 and 8 , where the differences of the terms in (26) for the pseudospin partners $\left(1 i_{11 / 2}, 2 g_{9 / 2}\right)$ and $\left(2 f_{5 / 2}, 3 p_{3 / 2}\right)$, respectively, are plotted. For states with higher angular momentum the magnitude of $\left\langle V_{\mathrm{PSO}}\right\rangle$ contribution for the energy splitting is higher, since it is roughly proportional to $2 \tilde{\ell}+1$, but there is a significant cancelation with the kinetic and $\langle\Sigma\rangle$ terms. A similar finding was already reported in [18], where it was pointed out that pseudospin-orbit coupling cannot be treated as a perturbative quantity. One the other hand, these figures show a clear correlation between the pseudospin-orbital term and the pseudospin energy splitting when the diffusivity and the depth of $\Sigma$ potential are varied. This feature does not depend on the particular pseudospin doublet under consideration.

The pseudospin-orbital term ( $\left.V_{\mathrm{PSO}}\right)$ comes from the coupling between the upper and the lower components. This term will vanish only when the upper component is zero (assuming a non-zero lower component) or when $\Sigma^{\prime}=0$, showing again that exact pseudospin symmetry is possible only when there are no bound states. This is not the case for the usual spin-orbit interaction generated by the term in the square brackets in Eq. (7). Here, this term will vanish when the lower component is zero, which corresponds to the non-relativistic limit.

From the results presented so far, we can conclude that the degeneracy of pseudospin levels is very much dependent on the shape of the nuclear mean field potential: the actual choice of the potential parameters, fitted to describe a nucleus, determines whether or not there is pseudospin symmetry. Furthermore, it is the strong cancelation presented in Figs. 6-8 that produces the quasi-degeneracy of pseudospin levels. The conclusion is that pseudospin symmetry is a dynamical symmetry in nuclei, in the sense of Arima's definition of dynamical symmetry [22]: (i) a symmetry of the Hamiltonian which is not geometrical in nature; or (ii) a ordered breaking symmetry from dynamical reasons. This dynamical nature of pseudospin symmetry is further stressed by the fact that pseudospin levels can cross each other. We observe also that the realization of pseudospin symmetry depends on the doublet in consideration, although the systematics is the same for all pseudospin partners.

\section{ISOSPIN ASYMMETRY OF PSEUDOSPIN SPLITTINGS}

We apply now this systematics to some nuclei studied in the literature, looking at the differences between neutron and proton spectra as far as pseudospin symmetry is concerned. 


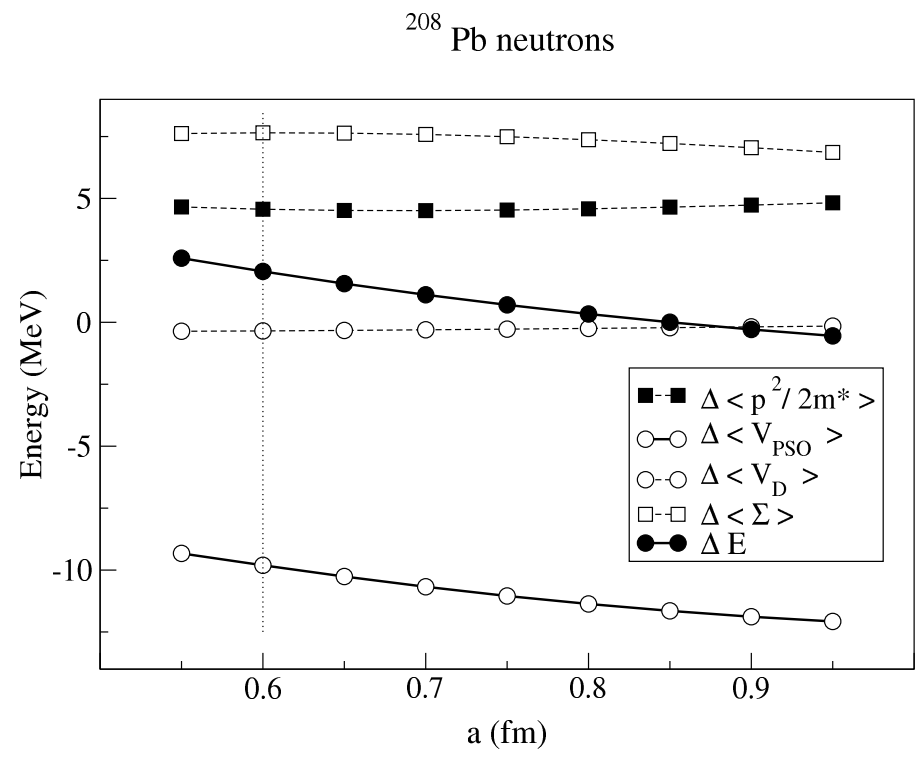

FIG. 7: Contribution of the terms $(27)$ - (30) to the energy splitting $\Delta E$ of the pseudospin partner $\left(1 i_{11 / 2}, 2 g_{9 / 2}\right)$ in ${ }^{208} \mathrm{~Pb}$ as a function of the diffuseness. The vertical line corresponds to the fitted Woods-Saxon parameters for ${ }^{208} \mathrm{~Pb}$ given in the caption of Fig. 1.

${ }^{208} \mathrm{~Pb}$ neutrons

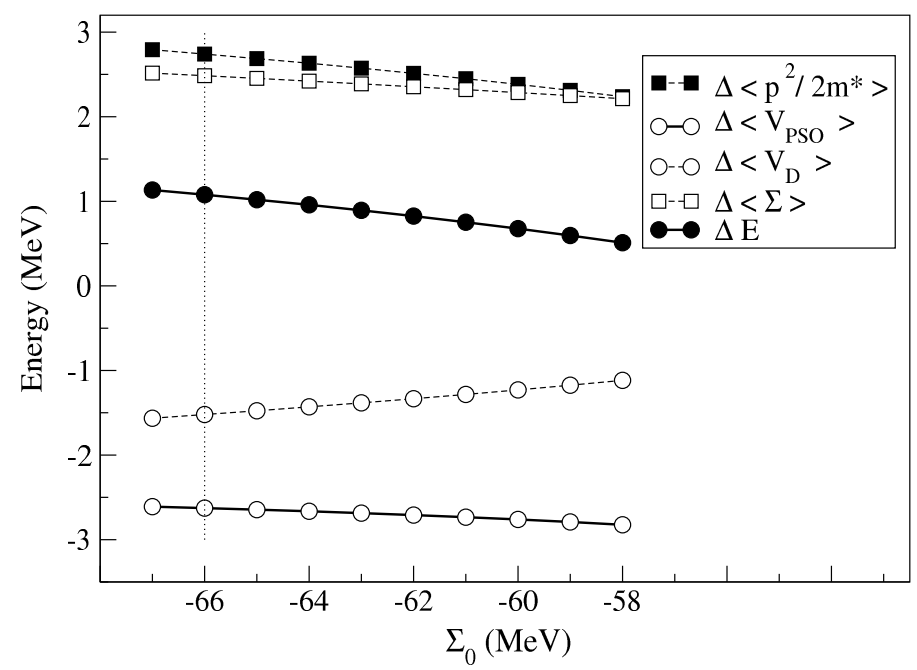

FIG. 8: Contribution of the terms $(27)-(30)$ to the energy splitting $\Delta E$ of the pseudospin partner $\left(2 f_{5 / 2}, 3 p_{3 / 2}\right)$ in ${ }^{208} \mathrm{~Pb}$ as a function of $\Sigma_{0}$. The vertical line corresponds to the fitted Woods-Saxon parameters for ${ }^{208} \mathrm{~Pb}$ given in the caption of Fig. 1 .

Recently, the pseudospin symmetry in Zr and Sn isotopes was investigated as a function of the number of nucleons [9]. The form of the $\Sigma$ potential as a function of the radial distance for such nuclei, as $A$ increases, is given in Ref. 28 , starting with ${ }^{100} \mathrm{Sn}$ and going up to ${ }^{170} \mathrm{Sn}$. This information on $\Sigma$ for Sn allows us to analyze the behavior for neutron pseudospin doublets going from $A=100$ to $A=170$. As $A$ increases, the modulus of the central depth, $\left|\Sigma_{0}\right|$, decreases and the surface diffuseness increases, both effects favoring the pseudospin symmetry, as shown in Figs. 2 and 3. However, as expected, the radius $R$ increases with $A$, which can (at least partially) offset the previous changes. This is what is observed in Ref. [9]: the energy splittings of deep neutron pseudospin partners decrease but those near the surface do not change sensibly as $A$ increases. The splitting for the partner $\left(1 h_{9 / 2}, 2 f_{7 / 2}\right)$ increases from a small negative value to a positive value, i.e., the levels cross each other. In [9] it is also observed that pseudospin splittings are smaller for levels close to the nucleus surface, even crossing each other. This phenomenon can also be observed in 
${ }^{208} \mathrm{~Pb}$, as one can see from the experimental energy values of the pseudospin partners $\left(2 f_{5 / 2}, 3 p_{3 / 2}\right)\left(1 i_{11 / 2}, 2 g_{9 / 2}\right)$ in Fig. 1. We do not expect our ansatz for the nuclear central potentials to explain the behavior of levels very near the nucleus surface. Such fine details are outside the scope of our work, which simply intends to establish a broad systematics for pseudospin splittings in nuclei.

For Zr isotopes, studied in refs. [8, 9], although the authors did not provide details on the potentials, again one sees that the pseudospin splitting in general decreases with $A$, with a somewhat irregular behavior for the partner $\left(1 g_{7 / 2}, 2 d_{5 / 2}\right)$. It is interesting to remark that the splitting for the doublet $\left(2 d_{3 / 2}, 3 s_{1 / 2}\right)$ decreases to the point of level crossing. Therefore, we can conclude that, as $A$ increases, there is a general trend for the pseudospin doublet partners to approach each other, towards realization of the symmetry.

The proton spectra of $\mathrm{Sn}$ isotopes was also studied in Ref. [28]. We observe that the central neutron depth, $\Sigma_{0}$, varies from about $-65 \mathrm{MeV}$ to $-54 \mathrm{MeV}$ and the surface diffuseness increases whereas the proton central depth, $\Sigma_{0}$, varies from $-50 \mathrm{MeV}$ to $-60 \mathrm{MeV}$ and the surface diffuseness slightly increases. The reason lies on the $\rho$ meson interaction, which for a neutron rich nuclei is repulsive for the neutrons and attractive for the protons, as explained by mean field model calculations [13]. The inclusion of this interaction, which is important in asymmetric nuclei, changes the vector part of the potential $V$ to

$$
V=V_{\omega}+V_{\rho}=V_{\omega} \pm \frac{g_{\rho}}{2} \rho_{0}
$$

with + and - signs for protons and neutrons respectively; $\rho_{0}$ is the time component of the $\rho$ field, which is proportional to the number of protons minus the number of neutrons, and $V_{\omega}$ comes from the vector-isoscalar $\omega$ meson. The increase of the parameters $R$ and $a$ with $A$ can be traced back to the known nuclear radius $A^{1 / 3}$ dependence and to the excess of neutrons on the surface (neutron skin effect). Hence, we may conclude that, for a given nucleus, the parameters $\Sigma_{0}, a$ and $R$ for protons and neutrons are different. Then, an isospin asymmetry in the pseudospin interaction is expected to take place in agreement with the analysis of the mean field nuclear parameters for protons and neutrons done in [5]. In particular, since $\rho_{0}$ is negative for heavy nuclei, the vector potential (31) is bigger (and, thus, $|\Sigma|$ is smaller) for neutrons than for protons. From our previous analysis, for a neutron rich nucleus, pseudospin symmetry for the neutron spectrum is favored, in agreement with the results presented by Lalazissis et al. [19].

The systematics discussed here seems to be quite general and a comment on how it affects the spin-orbit splittings is pertinent. Spin-orbit splittings are much less changed by variations of the central depth and surface diffuseness of the nuclear potential than pseudospin-orbit splittings. The reason is that to change substantially spin-orbit splittings one needs a significant relativistic content, i.e., a large lower component in the Dirac spinor [31]. The spin-orbit splitting is completely correlated with the nucleon effective mass [32]. From Eq. (13), we see that it will depend strongly on $\Delta$. This potential itself carries a quite large scale when compared with $\Sigma$ (around a factor ten). Therefore, the $\rho$ meson potential, $V_{\rho}$, generally about ten percent of $\Sigma$, becomes irrelevant compared to $\Delta$. This justifies why the spin-orbit interaction is roughly isospin symmetric, being almost the same for neutrons and for protons [28, 33].

On the contrary, the pseudospin splitting needs less relativistic content, i.e., a smaller lower component in order to change. This may explain why the non-relativistic analysis of Ref. [4] of the origin of the pseudospin symmetry, and manifested by Eq. (11), works well to explain the small pseudospin splitting. The pseudospin orbit interaction depends on $\Sigma$, as one can see from Eq. (12). This means that now $V_{\rho}$ cannot be neglected, since its values are comparable to those of $\Sigma$, causing sensibly different variations of the central depth for neutrons and protons, for which $V_{\rho}$ has different signs. This is one of the mechanisms generating the isospin asymmetry in the pseudospin-orbit interaction, as we have pointed out before. This can be seen in Fig. 9, where the energy splittings for the pseudospin partners $\left(1 h_{9 / 2}, 2 f_{7 / 2}\right),\left(2 f_{5 / 2}, 3 p_{3 / 2}\right)$ and for spin-orbit pairs $\left(2 f_{5 / 2}, 2 f_{7 / 2}\right),\left(1 h_{9 / 2}, 1 h_{11 / 2}\right)$ are plotted. Here we have just fixed the radius and diffusivity, using the values that fitted the neutron spectrum of ${ }^{208} \mathrm{~Pb}$, and let both $\Sigma_{0}$ and $\Delta_{0}$ change by the same amount, thereby simulating the increase of the vector potential $V$ that affects $\Sigma$ and $\Delta$ in the same way. It is clear that the splittings change much less for the spin-orbit doublets than for the pseudospin doublets. The magnitude of the change depends on the specific level one considers, but we checked that this kind of behavior occurs for deep levels. Of course, one should also consider the effect of the Coulomb potential for proton spectra, which has the opposite sign of $V_{\rho}$ for protons. However, we expect the effect of $V_{\rho}$ to be dominant for neutron rich nuclei.

\section{CONCLUSIONS}

We have studied, through a mean-field model calculation with Woods-Saxon potentials, the role played by the surface diffuseness, the radius, and the central depth of the sum of the standard vector and scalar nuclear potentials in the energy splittings of pseudospin partners, both for lead and calcium isotopes. This study allowed us to draw a systematics for the behavior of the pseudospin splitting as $a, R$ and $\Sigma_{0}$ vary. Such behavior was confirmed when 


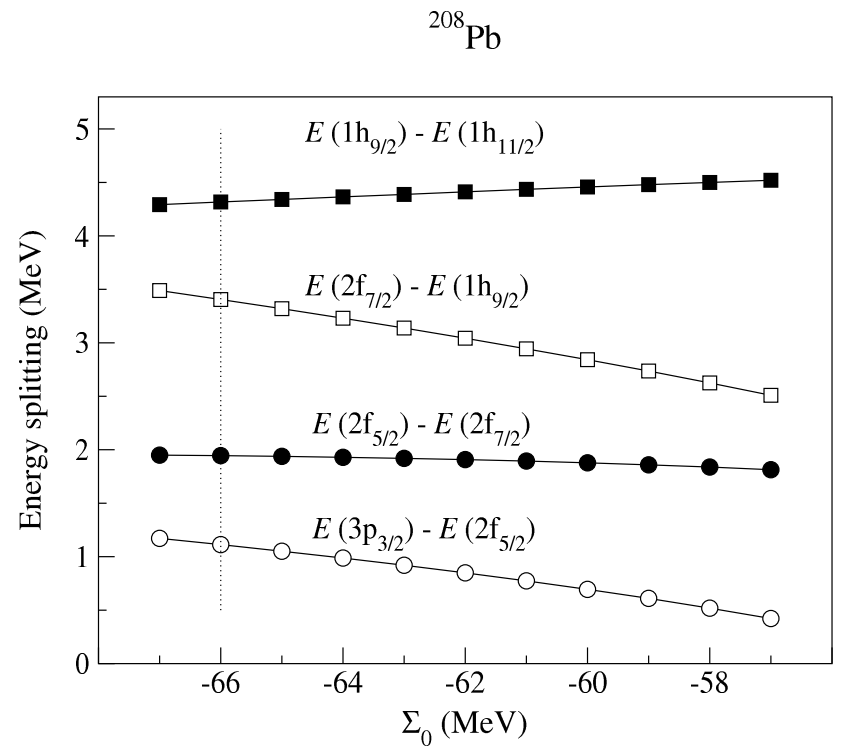

FIG. 9: Energy splitting in ${ }^{208} \mathrm{~Pb}$ as a function of the depth of the $\Sigma$ potential for the pseudospin partners $\left(1 h_{9 / 2}, 2 f_{7 / 2}\right)$, $\left(2 f_{5 / 2}, 3 p_{3 / 2}\right)$ (hollow symbols) and spin-orbit pairs $\left(2 f_{5 / 2}, 2 f_{7 / 2}\right),\left(1 h_{9 / 2}, 1 h_{11 / 2}\right)$ (filled symbols). The depth $\Delta_{0}$ varies from $639 \mathrm{MeV}$ to $649 \mathrm{MeV}$ so that only the vector potential changes. The vertical line corresponds to the fitted Woods-Saxon parameters for ${ }^{208} \mathrm{~Pb}$ given in the caption of Fig. 1 .

we applied the systematics to the Sn nuclei isotope chain, predicting the observed general trend towards degeneracy of pseudospin doublets. Based on our findings and in general features of the neutron and proton potentials, we explained the observed isospin asymmetry of pseudospin splittings. We were able to identify a pseudospin-orbital term and found that its contribution to the pseudospin energy splittings is large. The near degeneracy of pseudospin levels is obtained from a significant cancelation among the different terms in the Schroedinger-like equation for the lower component of the Dirac spinor. We also showed a clear correlation between the pseudospin-orbital term and the pseudospin energy splitting when the diffusivity and the depth of $\Sigma$ potential are varied. These findings led us to argue that pseudospin symmetry in nuclei is dynamic.

We believe that the systematics observed in our model calculation and the non-perturbative nature of the pseudospin-orbit term, which is larger than the energy splitting itself and needs to be canceled in order to produce the pseudospin quasi-degeneracy may help to understand how the pseudospin symmetry is dynamically broken in nuclei.

\section{Acknowledgments}

We acknowledge financial support from FCT (POCTI), Portugal, and from CNPq/ICCTI Brazilian-Portuguese scientific exchange program.

[1] K. T. Hecht and A. Adler, Nucl. Phys. A137, 129 (1969)

[2] A. Arima, M. Harvey, and K. Shimizu, Phys. Lett. B30, 517 (1969)

[3] P. Alberto, M. Fiolhais, M. Malheiro, A. Delfino and M. Chiapparini, Phys. Rev. Lett. 86, 5015 (2001)

[4] A. L. Blokhin, C. Bahri, and J. P. Draayer, Phys. Rev. Lett. 74, 4149 (1995)

[5] C. Bahri, J. P. Draayer, and S. A. Moszkowski, Phys. Rev. Lett. 68, 2133 (1992)

[6] O. Castaños, M. Moshinski, and C. Quesne, Phys. Lett. B277 238 (1992)

[7] J. N. Ginocchio, Phys. Rev. Lett. 78, 436 (1997); ibid, Phys. Rept. 315, 231 (1999)

[8] J. Meng, K. Sugawara-Tanabe, S. Yamaji, P. Ring, and A. Arima, Phys. Rev. C58, R628 (1998)

[9] J. Meng, K. Sugawara-Tanabe, S. Yamaji, and A. Arima, Phys. Rev. C59, 154 (1999)

[10] J. N. Ginocchio and A. Leviatan, Phys. Lett. B245, 1 (1998)

[11] B. Smith and L. J. Tassie, Ann. Phys. 65, 352 (1971) 
[12] J. S. Bell and H. Ruegg, Nucl. Phys. B98, 151 (1975)

[13] B. D. Serot and J. D. Walecka, The Relativistic Nuclear Many - Body Problem in Advances in Nuclear Physics, edited by J. W. Negele and E. Vogt, Vol. 16 (Plenum, New York, 1986); ibid, Int. J. Mod. Phys. E 6, 515 (1997) for a recent review

[14] R. J. Furnstahl and B. D. Serot Nucl. Phys. A673, 298 (2000)

[15] J. Zimany and S. A. Moszkowski, Phys. Rev. C42, 1416 (1990)

[16] A. Delfino, C. T. Coelho, and M. Malheiro, Phys. Rev. C51, 2188 (1995)

[17] A. Delfino, C. T. Coelho, and M. Malheiro, Phys. Lett. B345, 361 (1995)

[18] S. Marcos, L. N. Savushkin, M. López-Quelle and P. Ring, Phys. Rev. C62, 054309 (2000)

[19] G. A. Lalazissis, Y. K. Gambir, J. P. Maharana, C. S. Warke, and P. Ring, Phys. Rev. C58, R45 (1998)

[20] J. N. Ginocchio and D. G. Madland, Phys. Rev. C57, 1167 (1998)

[21] J. N. Ginocchio, Phys. Rev. Lett. 82, 4599 (1999)

[22] A. Arima, "Dynamical Symmetries and Nuclear Structure", preprint RIKEN-AF-NP-276

[23] K. Sugawara-Tanabe and A. Arima, Phys. Rev. C58, R3065 (1998)

[24] J. N. Ginocchio and A. Leviatan, Phys. Rev. Lett. 87, 072502 (2001)

[25] A. Leviatan and J. N. Ginocchio, nucl-th/0108016

[26] P. R. Page, T. Goldman, and J. N. Ginocchio, Phys. Rev. Lett. 86, 204 (2001)

[27] B. A. Nikolaus, T. Hoch, and D. G. Madland, Phys. Rev. C46, 1757 (1992)

[28] J. Meng and I. Tanihata, Nucl. Phys. A650, 176 (1999)

[29] R. J. Furnstahl, B. D. Serot and Hua-Bin Tang, Nucl. Phys. A615, 441 (1997)

[30] X. Campi and D. W. Sprung, Nucl. Phys. A399, 529 (1983)

[31] P. Alberto, M. Fiolhais, and M. Oliveira, Eur. J. Phys. 19, 553 (1998)

[32] R. J. Furnstahl, J. J. Rusnak, and B. D. Serot, Nucl. Phys. A632, 607 (1998)

[33] M. Chiapparini, A. Delfino, M. Malheiro, and A. Gattone, Z. Phys. A357, 47 (1997) 